\title{
Gastric Ulcer Complications after the Introduction of Proton Pump Inhibitors into Clinical Routine: 20-Year Experience
}

\author{
Friederike Eisnera \\ Diana Hermann $^{a} \quad$ Khaled Bajaeifer $^{\mathrm{b}} \quad$ Jörg Glatzle ${ }^{\mathrm{b}}$ \\ Markus A. Küperc \\ ${ }^{a}$ Department of General, Visceral and Transplant Surgery, University Hospital of Tübingen, Tübingen, Germany; \\ ${ }^{b}$ Department of General and Visceral Surgery, Medical Center of Konstanz, Konstanz, Germany; \\ ${ }^{c}$ Department of Traumatology and Reconstructive Surgery, BG Trauma Center, University of Tübingen, Tübingen, Germany
}

Alfred Königsrainer ${ }^{a}$

\section{Keywords}

Peptic ulcer disease - Ulcer hemorrhage .

Ulcer perforation - Ulcer excision - Surgical oversewing . Non-steroidal anti-inflammatory drugs, NSAIDs .

Helicobacter pylori

\section{Summary}

Background: The aim of this study was to analyze the admissions and the management of peptic ulcer disease (PUD) in a tertiary care surgical center. Methods: We evaluated the medical records of all patients admitted to the University Hospital of Tübingen, Germany, for treatment of PUD during 1989-2008. Patients were included into the study if the diagnosis was verified endoscopically or surgically. Annual number of admissions, length of hospitalization, mortality rate, age, rate of non-steroidal anti-inflammatory drugs (NSAIDs) and proton pump inhibitor (PPI) medication, rate of Helicobacter pylori infection, and complications of PUD and surgery performed were recorded. Data were analyzed by descriptive analyses, Pearson's chi-square test, and regression analysis. Results: This study included 614 admissions. The number of annual admissions $(31 \pm 12)$, the length of hospitalization ( $9 \pm 3$ days), and the mortality rate ( $5 \pm$ $4 \%$ per year) remained constant, whereas the age increased (1989: $52 \pm 14$ years vs. 2008: $67 \pm 16$ years). The rates of patients with $H$. pylori infection ( $47 \pm 28 \%$ per year), NSAIDs treatment ( $29 \pm 15 \%$ per year), and PPI treatment (31 $\pm 27 \%$ per year) remained constant. The most frequent PUD complication was hemorrhage ( $42 \pm 16 \%$ per year), followed by perforation ( $9 \pm 8 \%$ per year). During 1999-2008, more hemorrhages (125 vs. 121; $p<0.05$ ) and perforations (40 vs. $21 ; p<0.05$ ) were registered than during 1989-1998. The rate of emergency surgery increased from $70 \%$ during $1989-1998$ to $87 \%$ during 1999-2008. In contrast, elective surgery decreased from $21 \%$ during $1989-1998$ to $7 \%$ during $1999-2008$. Ulcer excision and oversewing was the most frequent surgical procedure performed (59\%), with decreasing rates of acid-reducing surgery. Conclusion: Despite recent advances in PUD management, ulcer hemorrhage and perforation remain a significant health burden and a surgical disease.

๑ 2017 S. Karger GmbH, Freiburg

\section{Introduction}

Peptic ulcer disease (PUD) remains a significant health burden and an important reason for hospitalization worldwide [1-3]. PUD results from an imbalance between the protective mechanisms of the gastrointestinal mucosa, such as mucus and bicarbonate secretion, and the harming impact of gastric acid and pepsin. This imbalance is predominantly associated with the use of non-steroidal anti-inflammatory drugs (NSAIDs) and acetylsalicylic acid (ASA) and/or Helicobacter pylori infection $[4,5]$. NSAIDs and $H$. pylori infection are independent risk factors and develop a synergistic effect on the development of gastric and duodenal mucosa damage, ulceration, and ulcer bleeding [4]. Gastric acid hypersecretion, psychological stress, nicotine and/or alcohol consumption, immunosuppressive medication, and age-related decline in prostaglandin levels are other important risk factors for the development of PUD $[4,6]$. There has been a dramatic change in the management of

\section{KARGER}

(c) 2017 S. Karger GmbH, Freiburg
Dr. med. Markus A. Küper 
PUD in recent decades: In the 1980s, H. pylori was identified as the most important cause of PUD [7]. Effective eradication therapies were established and provided previously not imaginable healing rates of PUD [8]. The important role of NSAIDs and ASA in PUD was recognized and initiated the search for alternative therapeutics and preventive strategies [4]. Nevertheless, the prescription and use of NSAIDs and ASA continues on a high level [9-11]. Today, histamine-2 receptor antagonists $\left(\mathrm{H}_{2} \mathrm{RA}\right)$ - launched in 1979 [12] and proton pump inhibitors (PPI) - launched in 1989 [13] - allow efficient inhibition of gastric acid secretion so that the so-called acid-reducing surgery is rarely needed. PPI provide healing rates of gastric and duodenal ulcer which even exceed the potential of $\mathrm{H}_{2}$ RA [14].

This study aims to analyze the impact of the recent changes in the management of PUD on the hospitalization and therapeutic strategies. Therefore, we evaluated all cases of PUD treated at a German tertiary care center in the first two decades after the introduction of PPI.

\section{Material and Methods}

\section{Patients}

The study was reviewed and approved by the local ethics committee of the University of Tübingen, Germany. This retrospective study analyzed the medical records of all patients admitted to the University Hospital of Tübingen, Germany, for gastric ulcer, intrapyloric ulcer, or a combination of gastric and duodenal ulcer between January 1989 and January 2008. Patients were included exclusively when the diagnosis was verified by endoscopy or surgery.

We analyzed the time trends regarding the following variables: number of admissions, length of hospital stay, age, overall mortality, presence of risk factors like NSAID and H. pylori, and PPI medication in the cohort. Time trends were analyzed in two ways: firstly, a continuous analysis over the 20-year period; secondly, a separate analysis of the two decades (1989-1998 and 1999-2008).

The following complications were recorded and analyzed in subgroups depending on the use of PPI: ulcer hemorrhage, ulcer perforation, ulcer penetration, and gastric outlet obstruction (GOO). Finally, we analyzed the surgical management of PUD complications in our cohort.

\section{Statistical Analysis}

Data are presented as mean \pm standard deviation (SD) if not stated otherwise. Time trends of the variables over the 20 -year period were analyzed using regression analysis to evaluate whether there is a correlation between the variables. The change of prevalence of the complications over the two decades as well as the correlation of PPI medication and the complications were analyzed using the Pearson's chi-square test. A p-value $<0.05$ was considered as significant. All analyses were done using Microsoft Excel (Microsoft Corporation, Redmond, WA, USA) software.

\section{Results}

\section{Demographic Data}

There had been a total of 518 patients with 614 admissions to the University Hospital of Tübingen, Germany, for gastric ulcer, intrapyloric ulcer, or a combination of gastric and duodenal ulcer during the years 1989-01/2008. The total number of patients ad-
Table 1. Demographic data of the cohort

\begin{tabular}{lc}
\hline Total admissions & 614 \\
1989-1998 & 334 \\
1999-01/2008 & 280 \\
Mean age, years (SD) & $62.5(17.5)$ \\
Gender & \\
$\quad$ Male (\% admissions) & $342(56)$ \\
$\quad$ Female (\% admissions) & $272(44)$ \\
Length of stay, days (SD) & $8.8(10.3)$ \\
Mortality (\% admissions) & $29(4.7)$ \\
\hline SD = Standard deviation. & \\
\hline
\end{tabular}

mitted for PUD was 334 in the first decade of observation (1989$1998)$ and 280 in the second decade (1999-01/2008). The mean age of the patients was $62.5 \pm 17.5$ years, with a female to male ratio of 1:1.3. The mean length of hospital stay was $8.8 \pm 10.3$ days. The overall mortality rate was $4.7 \%$ (table 1 ).

The mean number of cases treated annually was 31 , and we did not record a significant trend of the number of patients hospitalized for PUD. The mean age significantly increased from $51.8 \pm$ 14.3 years in 1989 to $67.5 \pm 16.1$ years in $2007 / 2008$. The mortality rate remained constant at $5 \%$ /year during the observation period. The length of hospital stay remained constant during the observed period (fig. 1-3).

\section{Complications of Peptic Ulcer Disease}

During the second decade of observation significantly more PUD complications (hemorrhage, perforation, ulcer penetration into adjacent organs, and GOO) were registered than during the first decade. In particular, more ulcer bleeding and perforation were registered in the second decade compared to the first decade (table 2).

The most frequent complication of PUD recorded during the observed period was ulcer bleeding (mean $42 \pm 16 \%$ /year), followed by ulcer perforation. The mean annual perforation rate was $9 \pm 8 \%$. Ulcer penetration and GOO were rarely observed. Between 1989 and 2008, the mean rate of ulcer penetration and of GOO was $2 \pm 2 \% /$ year and $1 \pm 3 \%$ /year, respectively (fig. 4 ).

\section{Risk Factors: Helicobacter pylori and NSAIDs}

The percentage of patients tested for $H$. pylori infection varied during the observation period. In the first years of observation the percentage of tested patients increased from $0 \%$ in 1989 to $39 \%$ in 1998. The mean rate of tested patients was $33 \pm 27 \% /$ year in the first decade of observation, with $48 \%$ of the tests being positive. In the following decade, the mean rate of tested patients increased to $65 \pm 14 \% /$ year, and $43 \%$ of the tests were positive. The annual rate of $H$. pylori infections peaked in 1992 and 1993 when $100 \%$ of the tests were positive. In 2008, none of the tested patients was positive for $H$. pylori infection.

During the observation period the rate of patients with NSAIDs at presentation varied. The mean rate of patients treated with NSAIDs at admission was $29 \pm 15 \%$ of the patients with known medication. The mean rate of patients hospitalized with unknown medication was $15 \pm 13 \%$. 


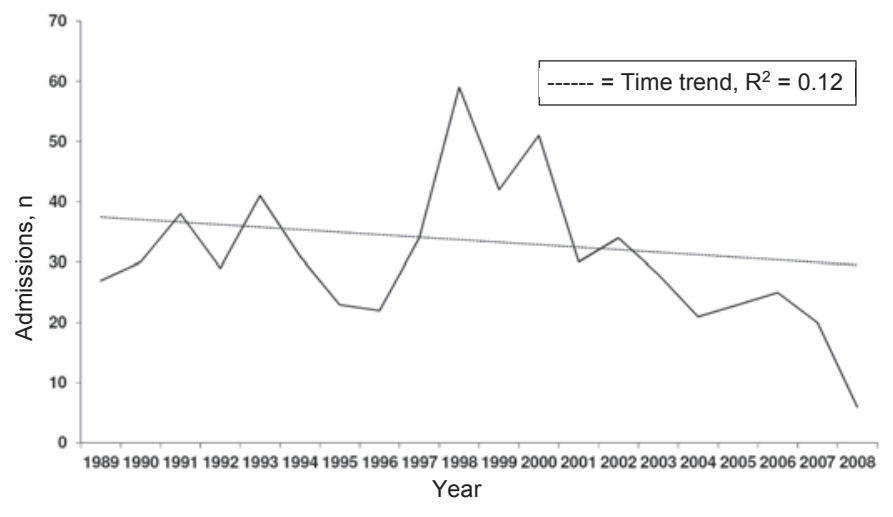

Fig. 1. Annual admission for peptic ulcer disease.

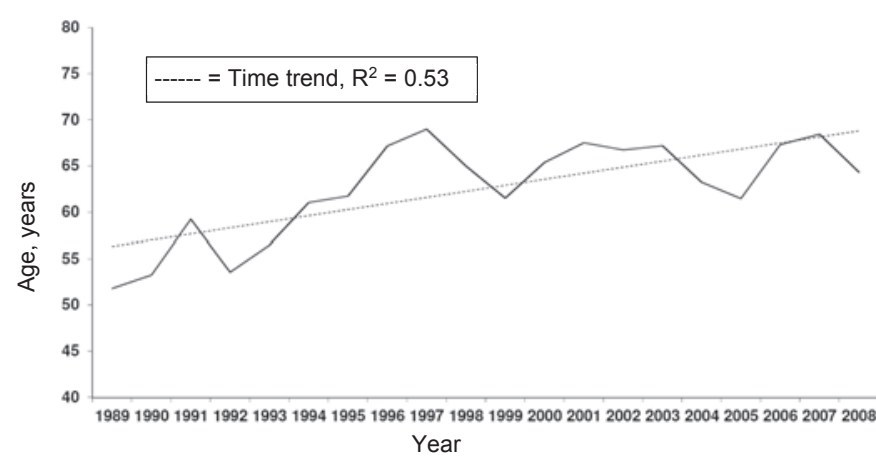

Fig. 2. Mean age of the patients hospitalized for peptic ulcer disease.

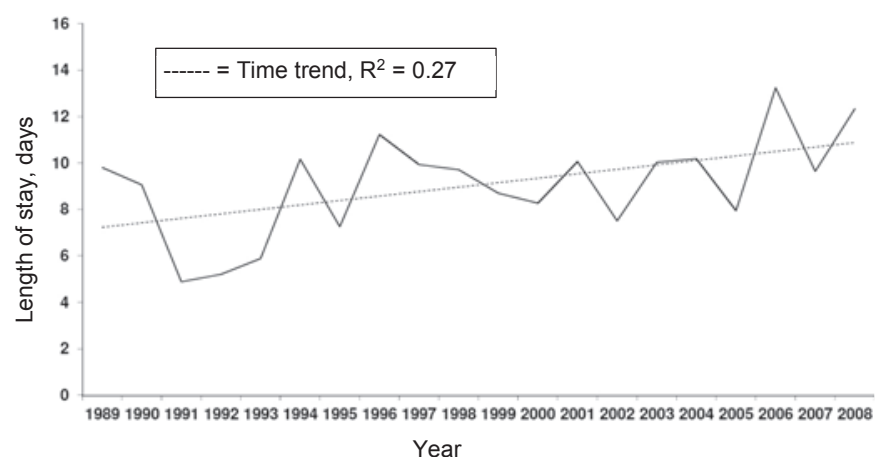

Fig. 3. Mean length of hospital stay for complicated peptic ulcer disease.

\section{Proton Pump Inhibitor Medication and Peptic Ulcer Disease Complications}

During the observation period the mean rate of the patients treated with PPI at admission was $31 \pm 27 \%$. Patients hospitalized with unknown medication were excluded from this calculation. We did not detect a significant correlation between PPI medication and PUD complications. Subgroup analysis, however, revealed that PPI medication was associated with a significantly lower rate of ulcer perforation in our cohort (table 3 ).

\section{Management of Ulcer Complications}

The importance of surgery in the treatment of PUD varied during the observation period. The operation rate of the hospitalized patients with complicated PUD was $28.2 \%$ and varied between $13 \%$ (1990) and 52\% (2004). A clear trend during the observation period was not noticeable. However, comparison of the two decades of observation (1989-1998 and 1999-2008) revealed that the rate of emergency surgery increased (70 vs. $87 \%$ ) while the rate of elective surgery decreased ( 21 vs. $7 \%$ ) (table 4 ).

The surgical strategy has also changed in the last 20 years: Overall, ulcer excision and oversewing was the most frequent surgical procedure performed during the observation period, with the rate of excision and oversewing increasing between the two decades (42 vs. $76 \%$ ). In contrast, the rate of acid-reducing surgery (resection and/or vagotomy) decreased ( 49 vs. $24 \%$ ). The rate of endoscopically treated hemorrhage remained unchanged between the first and second period of observation (27 vs. $28 \%$ ) (table 4).

\section{Discussion}

We analyzed the medical records of all patients treated for PUD at the University Hospital of Tübingen, Germany, from 1989 to 2008. We aimed to explore the effects of the recent advances in the medical treatment of PUD on the admissions and therapeutic strategies at our hospital.

We did not observe a significant decline of the patients hospitalized for PUD. However, the total number of patients admitted for PUD decreased from 334 in the first decade of observation (1989$1998)$ to 280 in the second decade (1999-01/2008). Several studies report a decrease of the incidence and prevalence of PUD as well as of the hospitalization rate for PUD since effective therapeutics for the reduction of gastric acid secretion and $H$. pylori eradication are available [1, 3, 15-19]. The position of our hospital as a tertiary care center with a supraregional, established surgical endoscopic unit explains that we did not register a significant decrease of annual admissions. Most of our patients are assigned to our hospital by practicing endoscopists or gastroenterologists if the possibilities of out-patient treatment are exhausted. Our analysis included only endoscopically or intraoperatively confirmed PUD.

The rate of PUD complications increased during the period of observation. We especially registered an increase of ulcer bleeding and perforation. Those findings are in line with data collected in the Netherlands [15], the USA [20], and England [17, 21, 22] during the 1990s. A decrease of PUD complications since 2000 is reported in Finnish [16], Norwegian [23], and US data [3]. In contrast to our study, which analyzed data from a single tertiary care center, those reports used aggregated data from hospital registers.

The general change of the age structure in western populations is reflected in the increase of the mean age of the patients admitted for PUD. During the observation period the mean age of our patients rose by almost 25\% (52 years in 1989 to 64 years in 01/2008). A similar dramatic ageing of the patients hospitalized for PUD in this period of time is reported in an English study [17]. In our cohort, the striking increase of the average age occurred in the 1990s. Two circumstances are frequently mentioned to explain those changes of the age structure; i.e., the declining prevalence of $H$. $p y$ - 
Fig. 4. Annual rates of ulcer complications.

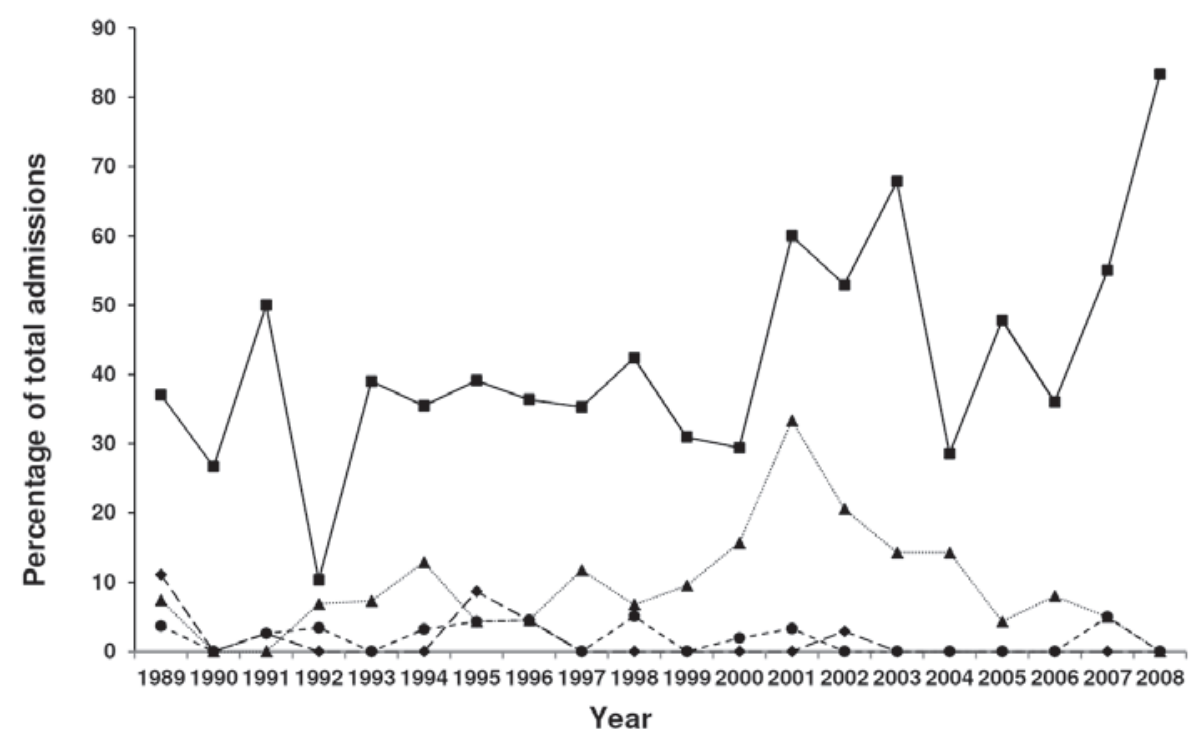

Table 2. Complications of peptic ulcer disease (PUD); over the last 20 years, there was an increase of PUD complications which were mainly due to more bleeding complications and perforations $s^{\mathrm{a}}$

\begin{tabular}{lccl}
\hline & $1989-1998$ & $1999-01 / 2008$ & p-value \\
\hline Total admissions & 334 & 280 & \\
\hline Total complications & $158(47.3)$ & $169(60.3)$ & 0.001 \\
$\quad$ Bleeding & $121(36.2)$ & $125(44.6)$ & 0.03 \\
Perforation & $21(6.3)$ & $40(14.3)$ & 0.001 \\
Penetration & $9(2.7)$ & $3(1.1)$ & 0.15 \\
GOO & $7(2.1)$ & $1(0.4)$ & 0.06 \\
\hline
\end{tabular}

${ }^{a}$ Data are total numbers with $\%$ of total admissions in brackets. $\mathrm{GOO}=$ Gastric outlet obstruction.

Table 3. Complications of peptic ulcer disease depending on proton pump inhibitor (PPI) medication; there was no difference in the total number of complications between patients who were set on PPI medication compared to no PPI medication; patients with PPI medication had significantly less perforations $\mathrm{s}^{\mathrm{a}}$

\begin{tabular}{lccc}
\hline & PPI & No PPI & p-value \\
\hline Total admissions & 198 & 308 & \\
\hline Total complications & $102(51.5)$ & $158(51.3)$ & 0.96 \\
$\quad$ Bleeding & $86(43.4)$ & $119(38.6)$ & 0.28 \\
Perforation & $13(6.6)$ & $37(12.0)$ & 0.04 \\
Penetration & $4(2.0)$ & $5(1.6)$ & 0.74 \\
GOO & $4(2.0)$ & $2(0.6)$ & 0.16 \\
\hline
\end{tabular}

${ }^{\mathrm{a} D a t a}$ are total numbers with $\%$ of total admissions in brackets. $\mathrm{GOO}=$ Gastric outlet obstruction

lori infection and the successes of eradication therapy decreased the hospitalization rate especially in the younger population. In contrast, an augmented use of low-dose aspirin for the prophylaxis of cardiovascular disorders and a widespread use of long-term NSAIDs medication increased PUD in the elderly [19, 24]. Maybe an increasing use of COX-2 inhibitors and the prophylactic administration of PPI might impede this trend in the next years. The higher age of PUD patients might also explain the increase of ulcer hemorrhage and perforation registered in our cohort. Amongst older individuals, antiplatelet agents and anticoagulants are more common than amongst the younger population. In addition, elderly patients often use analgesics permanently, and these potentially mask the body's danger pain signal. Those circumstances promote that ulcer bleeding or perforation might be the first symptom of PUD. Bardhan and Royston [17] recorded a clear relationship between individuals of 65 years and above and hemorrhage as the first manifestation of PUD in an English cohort.

The rate of patients operated for PUD varied during the observation period. Comparison of the two observed decades (1989-1998 and 1999-01/2008) revealed an increase of patients requiring emergency surgery and a remarkable decline of elective surgery. Other authors registered a similar decrease in elective PUD surgery [3, 17]. Although the modern medical management of PUD virtually replaced the so-called acid-reducing surgery (which consists of antral resection and/or vagotomy), the decrease of elective PUD surgery started long before the introduction of $\mathrm{H}_{2} \mathrm{RA}$ and PPI. Declining rates were already registered in Sweden during the 1960s [25], in England in the 1970s [22], and in Finland in the 1980s [12]. The authors of these studies speculate extensively about the reasons for this trend. Probably the combination of several circumstances accounts for the early decrease of elective PUD surgery: Advances in fiberoptic endoscopy allowed to detect malignancy without surgery and to distinguish non-ulcer dyspepsia. Surgical strategies altered over the years and indications changed. The socioeconomic standing might impact the course of PUD as well [12, 17, 25, 26].

Ulcer excision and oversewing was the most frequent surgical procedure in our cohort. The rate of patients requiring this procedure markedly increased in the second decade of observation (1989-1998: 45\% vs. 1999-01/2008: 76\%). This corresponds to the 
Table 4. Management of ulcer complications in the two decades of observation

\begin{tabular}{lccl}
\hline & $1989-1998$ & $1999-01 / 2008$ & p-value \\
\hline Total admissions & 334 & 280 & \\
\hline Bleeding ulcer (\% admissions) & $121(36.2)$ & $125(44.6)$ & 0.03 \\
$\quad$ Endoscopic hemostasis (\% bleeding) & $92(76.0)$ & $81(64.8)$ & 0.05 \\
\hline Surgery (\% admissions) & $94(28.1)$ & $79(28.2)$ & 0.99 \\
$\quad$ Emergency surgery (\% surgery) & $66(70.2)$ & $69(87.3)$ & 0.007 \\
Delayed surgery (\% surgery) & $8(8.5)$ & $4(5.1)$ & 0.37 \\
Elective surgery (\% surgery) & $20(21.3)$ & $6(7.6)$ & 0.01 \\
\hline Type of surgery & & & \\
Excision/Oversewing (\% surgery) & $42(44.7)$ & $60(75.9)$ & $<0.001$ \\
Acid-reducing surgery (\% surgery) & $46(48.9)$ & $19(24.1)$ & 0.001 \\
Other (\% surgery) & $6(6.4)$ & $2(2.5)$ & 0.23 \\
\hline${ }^{a}$ Acid-reducing surgery = Gastric resection and/or vagotomy. & & \\
\hline
\end{tabular}

increased rate of ulcer perforation (1989-1998: 6\% vs. 199901/2008: 14\%). The augmented rate of patients presenting with ulcer bleeding in the second decade of observation (1989-1998: $36 \%$ vs. $1999-01 / 2008$ : $45 \%$ ) also seems to contribute to the increase of the excision and oversewing rate. The rate of ulcer hemorrhage treated by endoscopy tended to decrease in the second decade of observation (1989-1998: 76\% vs. 1999-01/2008: 65\%). This might be due to an increased rate of hardly controllable hemorrhages.

This study has limitations that should be considered. First, as a retrospective, single-institution study analyzing data from a tertiary care surgical center, our study is subject to selection bias. Our data are not representative for a certain population and do not allow conclusions regarding overall incidence and prevalence. Second, most of our patients presented as an emergency. Comorbidities and medication were incompletely registered in many cases, and a considerable number of patients were not tested for $H$. pylori infection during the hospital stay due to the emergency situation. Therefore, our data permit only limited conclusions regarding morbidity and the impact of PPI medication itself. Nevertheless, our study provides insights into the characteristics of PUD and the challenges the medical staffs should be aware of. The importance of emergency surgery and endoscopy remains on a high level, and a substantial part of the patients presents during the weekend, evening, or night time when the staff is reduced [23]. Because of the striking decrease of elective PUD surgery, several specific surgical procedures almost disappeared but possibly have to be performed in an emergency situation. This causes substantial challenges during surgical formation.

\section{Conclusion}

In summary, the general decline of PUD incidence and prevalence also affects the number of patients admitted to our tertiary care center. However, the rate of PUD complications is increasing, and especially the rates of severe hemorrhage and perforation requiring surgical intervention increased in our cohort. We registered a considerable ageing of the patients hospitalized for PUD. Elective PUD surgery loses importance whereas the rate of patients requiring emergency surgery even increased in our cohort.

\section{Acknowledgment}

Author contribution: F. Eisner, D. Hermann, K. Bajaeifer, J. Glatzle, A. Königsrainer, and M.A. Küper substantially contributed to the conception and design of the work and to the acquisition, analysis, and interpretation of data. The authors drafted the manuscript and revised its intellectual content critically. All authors approved the final version of the article to be published.

\section{Disclosure Statement}

To the best of our knowledge, no conflict of interest exists.

\section{References}

1 Sung JJY, Kuipers EJ, El-Serag HB: Systematic review: the global incidence and prevalence of peptic ulcer disease. Aliment Pharmacol Ther 2009;29:938-946.

2 Søreide K, Thorsen K, Søreide JA: Strategies to improve the outcome of emergency surgery for perforated peptic ulcer. Br J Surg 2014;101:e51-64.
3 Wang YR, Richter JE, Dempsey DT: Trends and outcomes of hospitalizations for peptic ulcer disease in the United States, 1993 to 2006. Ann Surg 2010;251:51-58.

4 Yuan Y, Padol IT, Hunt RH: Peptic ulcer disease today. Nat Clin Pract Gastroenterol Hepatol 2006;3:80-89.
5 Ramakrishnan K, Salinas RC: Peptic ulcer disease. Am Fam Physician 2007;76:1005-1012.

6 Osefo N, Ito T, Jensen RT: Gastric acid hypersecretory states: recent insights and advances. Curr Gastroenterol Rep 2009;11:433-441. 
7 Marshall BJ, Warren JR: Unidentified curved bacilli in the stomach of patients with gastritis and peptic ulceration. Lancet 1984;1:1311-1315.

8 Leodolter A, Kulig M, Brasch H, Meyer-Sabellek W, Willich SN, Malfertheiner P: A meta-analysis comparing eradication, healing and relapse rates in patients with Helicobacter pylori-associated gastric or duodenal ulcer. Aliment Pharmacol Ther 2001;15:1949-1958.

9 Sarganas G, Buttery AK, Zhuang W, Wolf I-K, Grams D, Rosario AS, Scheidt-Nave C, Knopf H: Prevalence, trends, patterns and associations of analgesic use in Germany. BMC Pharmacol Toxicol 2015;16:28.

10 Dale O, Borchgrevink PC, Fredheim OMS, Mahic M, Romundstad P, Skurtveit S: Prevalence of use of nonprescription analgesics in the Norwegian HUNT3 population: impact of gender, age, exercise and prescription of opioids. BMC Public Health 2015;15:461.

11 Breivik H, Collett B, Ventafridda V, Cohen R, Gallacher D: Survey of chronic pain in Europe: prevalence, impact on daily life, and treatment. Eur J Pain Lond Engl 2006;10:287-333.

12 Paimela H, Tuompo PK, Peräkyl T, Saario I, Höckerstedt K, Kivilaakso E: Peptic ulcer surgery during the H2-receptor antagonist era: a population-based epidemiological study of ulcer surgery in Helsinki from 1972 to 1987. Br J Surg 1991;78:28-31.

13 Mössner J: The indications, applications, and risks of proton pump inhibitors. Dtsch Ärztebl Int 2016;113. $477-483$.
14 Salas M, Ward A, Caro J: Are proton pump inhibitors the first choice for acute treatment of gastric ulcers? A meta-analysis of randomized clinical trials. BMC Gastroenterol 2002;2:17.

15 Post PN, Kuipers EJ, Meijer GA: Declining incidence of peptic ulcer but not of its complications: a nationwide study in The Netherlands. Aliment Pharmacol Ther 2006;23:1587-1593.

16 Malmi H, Kautiainen H, Virta LJ, Färkkilä N, Koskenpato J, Färkkilä MA: Incidence and complications of peptic ulcer disease requiring hospitalisation have markedly decreased in Finland. Aliment Pharmacol Ther 2014;39:496-506

17 Bardhan KD, Royston C: Time, change and peptic ulcer disease in Rotherham, UK. Dig Liver Dis 2008; 40:540-546.

18 Kang J-Y, Tinto A, Higham J, Majeed A: Peptic ulceration in general practice in England and Wales 199498: period prevalence and drug management. Aliment Pharmacol Ther 2002;16:1067-1074.

19 Lassen A, Hallas J, Schaffalitzky de Muckadell OB: Complicated and uncomplicated peptic ulcers in a Danish county 1993-2002: a population-based cohort study. Am J Gastroenterol 2006;101:945-953.
20 Lewis JD, Bilker WB, Brensinger C, Farrar JT, Strom BL: Hospitalization and mortality rates from peptic ulcer disease and GI bleeding in the 1990s: relationship to sales of nonsteroidal anti-inflammatory drugs and acid suppression medications. Am J Gastroenterol 2002;97:2540-2549.

21 Higham J, Kang J-Y, Majeed A: Recent trends in admissions and mortality due to peptic ulcer in England: increasing frequency of haemorrhage among older subjects. Gut 2002;50:460-464.

22 Bardhan KD, Williamson M, Royston C, Lyon C: Admission rates for peptic ulcer in the Trent Region, UK, 1972-2000. Changing pattern, a changing disease? Dig Liver Dis 2004;36:577-588.

23 Thorsen K, Søreide JA, Kvaløy JT, Glomsaker T, Søreide K: Epidemiology of perforated peptic ulcer age- and gender-adjusted analysis of incidence and mortality. World J Gastroenterol 2013;19:347-354.

24 Kang JY, Majeed A: Peptic ulcer - time trends at the turn of the millennium. Dig Liver Dis 2004;36:570571.

5 Gustavsson S, Nyrén O: Time trends in peptic ulcer surgery, 1956 to 1986. A nation-wide survey in Sweden. Ann Surg 1989;210:704-709.

26 Smith JW, Mathis T, Benns MV, Franklin GA, Harbrecht BG, Larson G: Socioeconomic disparities in the operative management of peptic ulcer disease. Surgery 2013;154:672-678; discussion 678-679. 